\title{
Neoplasias espontâneas em ratos Wistar de um centro de criação de animais de laboratório do Estado do Rio de Janeiro, Brasil
}

\author{
Spontaneous neoplasms in Wistar rats from a center for laboratory animal breeding of the Rio de \\ Janeiro state, Brazil
}

\author{
Marcele Nogueira de Sousa Trotte ${ }^{\mathrm{I} *}$ Rodrigo Caldas Menezes $^{\mathrm{II}}$ Rogerio Tortelly $^{\mathrm{I}}$
}

\section{RESUMO}

Este estudo foi realizado com o objetivo de verificar a prevalência e as características anatomopatológicas de neoplasias espontâneas encontradas em ratos Wistar, provenientes do biotério do Centro de Criação de Animais de Laboratório, FIOCRUZ, Rio de Janeiro. Foram necropsiados 1.964 ratos Wistar, no período entre agosto de 2002 e janeiro de 2007, após eutanásia em câmara de gás carbônico. Amostras teciduais foram colhidas, fixadas em formol tamponado a $10 \%$ e processadas pelas técnicas habituais para inclusão em parafina. Foram realizados 38 diagnósticos de neoplasmas, sendo 36 ratos acometidos, o que representa uma prevalência de $1,8 \%$. Dos 36 animais, apenas seis $(16,7 \%)$ eram machos e um (2,8\%) jovem. As neoplasias diagnosticadas com as respectivas ocorrências foram: carcinoma mamário (42,1\%), fibroadenoma mamário (13,2\%), hemangiossarcoma (7,9\%), adenoma da glândula hipófise (7,9\%), adenoma mamário (5,3\%), fibrossarcoma $(5,3 \%)$, adenocarcinoma papilífero pulmonar primário (2,6\%), epitelioma sebáceo (2,6\%), ganglioneuroblastoma na glândula adrenal (2,6\%), leiomioma uterino (2,6\%), mesotelioma $(2,6 \%)$, osteossarcoma osteoblástico $(2,6 \%)$ e papiloma (2,6\%). No presente estudo a prevalência de neoplasias espontâneas foi bastante inferior à encontrada em estudos similares, com predomínio dos tumores malignos. O epitelioma sebáceo e os carcinomas mamários mucinoso e simples císticopapilar com ocorrência espontânea foram descritos pela primeira vez em ratos Wistar.

Palavras-chave: animal de laboratório, oncologia, patologia, prevalência, Rattus norvegicus

\section{ABSTRACT}

The purpose of the present study was to check the prevalence and the pathologic features of spontaneous neoplasms found in Wistar rat, from the production colonies at the Center for Laboratory Animal Breeding, FIOCRUZ, Rio de Janeiro. One thousand nine hundred and sixty four Wistar rats were submitted to necropsy in the period between August 2002 and January 2007, after euthanasia in CO chamber. Tissue samples were collected, fixed in $10 \%$ buffered formalin and processed by the usual techniques for inclusion in paraffin. Thirty eight neoplasms were diagnoses and 36 rats had tumors, representing a prevalence of $1.8 \%$. Among the 36 animals, only six (16.7\%) were male and one (2.8\%) young. The neoplasms were diagnosed with the following occurrences: mammary carcinoma (42.1\%), mammary fibroadenoma $(13.2 \%)$, hemangiosarcoma $(7.9 \%)$, adenoma of the pituitary gland (7.9\%), mammary adenoma (5.3\%), fibrosarcoma (5.3\%), primary papillary pulmonary adenocarcinoma (2.6\%), sebaceous epithelioma (2.6\%), ganglioneuroblastoma in the adrenal gland (2.6\%), leiomyoma of the uterus (2.6\%), mesothelioma (2.6\%), osteosarcoma osteoblastic (2.6\%), papilloma (2.6\%). In this study the prevalence of spontaneous neoplasms was lower than the ones found in similar studies and the malignant tumors predominated. The sebaceous epithelioma and also the mucinous and simple papillary-cystic mammary carcinomas with spontaneous occurrence were described in Wistar rats for the first time.

Key words: laboratory animal, oncology, pathology, prevalence, Rattus norvegicus.

\section{INTRODUÇÃO}

Estudos sobre a ocorrência de neoplasmas espontâneos em animais de laboratório levaram ao desenvolvimento de modelos animais e forneceram dados de base para os trabalhos de carcinogênese experimental (KOHN \& BARTHOLD, 1984).

IDepartamento de Patologia e Clínica Veterinária, Faculdade de Veterinária, Universidade Federal Fluminense (UFF). Rua vital Brazil Filho, 64, 24230-340, Santa Rosa, Niterói, RJ, Brasil. E-mail: marceletrotte@vm.uff.br. *Autor para correspondência.

IIServiço de Zoonoses, Instituto de Pesquisa Clínica Evandro Chagas, Fiocruz, Rio de Janeiro, RJ, Brasil. 
Nos ratos Wistar, o adenoma da glândula hipófise e o fibroadenoma mamário compreendem os tumores de maior ocorrência, com 50\% e 23\%, respectivamente (POTERACKI \& WALSH, 1998). Com ocorrência entre 6 e 13\%, já foram relatados casos de feocromocitoma benigno da glândula adrenal, adenoma de célula $C$ da glândula tireóide, adenocarcinoma uterino (BOMHARD \& RINKE, 1994), adenoma do córtex da glândula adrenal, adenocarcinoma mamário (WALSH \& POTERACKI, 1994), adenoma de célula acinar do pâncreas, queratoacantoma, tumor de célula intersticial testicular e timoma benigno (POTERACKI \& WALSH, 1998).

Neoplasmas de alta freqüência em animais são valiosos como modelos de estudos de carcinogênese e para outras áreas da oncologia, já que estes têm similaridade com os neoplasmas encontrados em seres humanos (PECKHAM, 1980). Apesar de a importância do conhecimento da ocorrência e dos tipos de neoplasias nas diferentes espécies de animais de laboratório, não há levantamento em biotérios brasileiros sobre essas doenças. O diagnóstico conclusivo é importante no diferencial com outras enfermidades nesses animais. Além disso, esses animais estão sendo comercializados e criados como animais de estimação. Portanto, o presente estudo teve por objetivo verificar a prevalência e as características anatomopatológicas de neoplasias espontâneas encontradas em ratos Wistar, provenientes do biotério do Centro de Criação de Animais de Laboratório, FIOCRUZ, Rio de Janeiro, Brasil. O estudo também trará contribuições para o diagnóstico dessas enfermidades na clínica desses animais.

\section{MATERIAL E MÉTODOS}

No período de agosto de 2002 a janeiro de 2007, foram estudados 1.964 ratos (Rattus norvegicus) da linhagem Wistar, livres de germes patogênicos específicos (SPF) e convencionais, criados e mantidos no Centro de Criação de Animais de Laboratório (CECAL), FIOCRUZ, Rio de Janeiro, RJ. Os animais eram mantidos em gaiolas de polipropileno, com cama de maravalha autoclavada e tampa de aço inox, em salas à temperatura de $21 \mathrm{a} 24^{\circ} \mathrm{C}$, com $10 \mathrm{a} 15$ trocas de ar por hora, umidade de $45 \%$ a $55 \%$ e ciclo de luz de 12 horas. A água, autoclavada para os animais SPF ou filtrada para os convencionais, era fornecida em bebedouros autoclavados e trocada a cada dois dias. A ração era fornecida ad libitum e formulada para roedores. A Nuvital CR- $1^{\circledR}$ autoclavável era fornecida aos animais SPF e Nuvital CR- $1{ }^{\circledR}$ para os convencionais.
Os animais foram submetidos à eutanásia em câmara de gás carbônico e foram necropsiados para monitoramento sanitário no Departamento de Controle da Qualidade Animal do CECAL. Entre esses animais, 738 eram machos, 884 fêmeas e 342 sem informações sobre o sexo. Além disso, 863 deles eram adultos (com idade entre três e 18 meses), 142 jovens (com idade entre 21 dias e dois meses) e 959 sem idade informada. Dos 1.964 ratos analisados, 824 eram convencionais e 1.140SPF.

Nas necropsias, amostras teciduais foram colhidas e imediatamente fixadas em solução de formol tamponado a $10 \%$. Posteriormente, os tecidos foram encaminhados ao Serviço de Anatomia Patológica Veterinária Professor Jefferson Andrade dos Santos, da Universidade Federal Fluminense, onde foram processados pelas técnicas habituais para inclusão em parafina e corados pela hematoxilina-eosina (HE). A coloração Fucsina Ácida-Van Gieson foi utilizada especificamente em caso de suspeita de leiomioma uterino. As lâminas de histopatologia foram analisadas em microscópico óptico (Olympus Bx 41) e as fotomicrografias foram obtidas a partir de uma câmera digital acoplada ao microscópio.

Os neoplasmas foram diagnosticados de acordo com a classificação histológica dos tumores nos animais, seguindo os critérios da Organização Mundial de Saúde (DUNGWORTH et al., 1999; GOLDSCHMIDT et al., 1998; HENDRICK et al.; 1998; KENNEDY et al., 1998; KOESTNER et al., 1999; MISDORP et al., 1999; SLAYTER et al., 1994).

Os resultados foram tabulados e foi determinada a prevalência de neoplasias em ratos Wistar e a freqüência em relação à classificação histológica dos tumores, ao sexo e à idade dos animais com neoformação. Dos animais sem informação sobre sexo e idade, nenhum apresentou neoplasia.

\section{RESULTADOS E DISCUSSÃO}

Trinta e seis ratos Wistar apresentaram neoplasias, representando uma prevalência de 1,8\%. Essa casuística é bastante inferior à relatada por BOMHARD \& RINKE (1994), WALSH \& POTERACKI (1994) e POTERACKI \& WALSH (1998), que encontraram prevalência de $46 \%$, 76\% e $83 \%$, respectivamente. Acredita-se que um dos motivos para a menor prevalência de neoplasias neste trabalho se deve à idade dos animais, visto que foram estudados animais de até 18 meses, já nos trabalhos supracitados, os indivíduos eram mais velhos, com idades entre $18 \mathrm{e}$ 26 meses e, portanto, mais propensos à ocorrência de tumores. Outros fatores também podem estar 
relacionados, tais como: população estudada, período de estudo, localização geográfica e nutrição dos animais (HARKNESS \& WAGNER, 1993). Vale ressaltar que o número de diagnósticos de neoplasmas foi maior (38) que o número de animais com neoplasma (36) em função de dois animais terem apresentado mais de um tumor simultaneamente. A ocorrência de neoplasias foi maior em fêmeas (83,3\%) e nos adultos (97,2\%), o que está de acordo com os demais estudos (BOMHARD \& RINKE, 1994; WALSH \& POTERACKI, 1994; POTERACKI \& WALSH, 1998).

Dos animais com neoplasias, apenas um era jovem, com dois meses de idade, e esse indivíduo apresentou carcinoma tubular mamário. No que se refere à classificação sanitária, dos animais com tumores, somente quatro eram convencionais. Na tabela 1 , encontra-se a ocorrência dos tipos de neoplasias nos ratos Wistar, conforme seu sexo.

Neste estudo os tumores malignos foram mais prevalentes, sendo o carcinoma mamário o de maior ocorrência (42,1\%). Esses resultados diferem dos encontrados por BOMHARD \& RINKE (1994), WALSH \& POTERACKI (1994) e POTERACKI \& WALSH (1998), em que o tumor de maior ocorrência foi o adenoma da glândula hipófise (38,6 e 54,0\%).

O segundo tumor mais freqüente no presente trabalho foi o fibroadenoma mamário (13,9\%), concordando com os resultados de WALSH \& POTERACKI (1994) e POTERACKI \& WALSH(1998), e discordando dos resultados de BOMHARD \& RINKE (1994), com 17\%, 23\% e 4\%, respectivamente.

Tabela 1 - Tipos de neoplasias em ratos Wistar, provenientes do Centro de Criação de Animais de Laboratório (CECAL), apresentados de acordo com o sexo dos animais (período de agosto de 2002 a janeiro de 2007).

\begin{tabular}{|c|c|c|c|}
\hline Grupos e tipos de neoplasias & $\begin{array}{l}\text { №. de neoplasias em } \\
\text { machos/ ocorrência }\end{array}$ & $\begin{array}{l}\text { №. de neoplasias em } \\
\text { fêmeas/ ocorrência }\end{array}$ & $\begin{array}{c}\text { №. total de neoplasias/ } \\
\text { ocorrência }\end{array}$ \\
\hline Neoplasias da glândula mamária & & & $23 / 60,5 \%$ \\
\hline Carcinoma simples tubular & - & $9 / 23,7 \%$ & $9 / 23,7 \%$ \\
\hline Carcinoma mucinoso & - & $5^{\mathrm{b}} / 13,2 \%$ & $5 / 13,2 \%$ \\
\hline Fibroadenoma & - & $5 / 13,2 \%$ & $5 / 13,2 \%$ \\
\hline Adenoma & - & $2 / 5,3 \%$ & $2 / 5,3 \%$ \\
\hline Carcinoma simples cístico-papilar & - & $2 / 5,3 \%$ & $2 / 5,3 \%$ \\
\hline Neoplasias mesenquimatosas da pele e dos tecidos moles & & & $6 / 15,8 \%$ \\
\hline Hemangiossarcoma & $2 / 5,3 \%$ & $1 / 2,6 \%$ & $3 / 7,9 \%$ \\
\hline Fibrossarcoma & - & $2 / 5,3 \%$ & $2 / 5,3 \%$ \\
\hline Mesotelioma & $1^{\mathrm{a}} / 2,6 \%$ & - & $1 / 2,6 \%$ \\
\hline Neoplasias das glândulas endócrinas & & & $4 / 10,5 \%$ \\
\hline \multicolumn{4}{|l|}{ Glândula hipófise } \\
\hline Adenoma & $2 / 5,3 \%$ & $1^{\mathrm{b}} / 2,6 \%$ & $3 / 7,9 \%$ \\
\hline \multicolumn{4}{|l|}{ Glândula adrenal } \\
\hline Ganglioneuroblastoma & $1^{a} / 2,6 \%$ & - & $1 / 2,6 \%$ \\
\hline Neoplasias epiteliais e melanocíticas da pele & & & $2 / 5,3 \%$ \\
\hline Epitelioma sebáceo & - & $1 / 2,6 \%$ & $1 / 2,6 \%$ \\
\hline Papiloma & $1 / 2,6 \%$ & - & $1 / 2,6 \%$ \\
\hline Neoplasias do osso e articulações & & & $1 / 2,6 \%$ \\
\hline Osteossarcoma osteoblástico & - & $1 / 2,6 \%$ & $1 / 2,6 \%$ \\
\hline Neoplasias do sistema genital & & & $1 / 2,6 \%$ \\
\hline Leiomioma & - & $1 / 2,6 \%$ & $1 / 2,6 \%$ \\
\hline Neoplasias do sistema respiratório & & & $1 / 2,6 \%$ \\
\hline Adenocarcinoma papilífero pulmonar primário & - & $1 / 2,6 \%$ & $1 / 2,6 \%$ \\
\hline
\end{tabular}

${ }^{\text {a } U m ~ m a c h o ~ a p r e s e n t o u ~ d o i s ~ t i p o s ~ d e ~ n e o p l a s i a s . ~}$

${ }^{\mathrm{b}}$ Uma fêmea apresentou dois tipos de neoplasias. 
Nas fêmeas, o carcinoma mamário foi a neoplasia mais freqüente e, nos machos, o hemangiossarcoma e o adenoma da glândula hipófise, diferente dos achados de BOMHARD \& RINKE (1994), WALSH \& POTERACKI (1994) e POTERACKI \& WALSH (1998), nos quais o adenoma da glândula hipófise foi o tumor de maior ocorrência em ambos os sexos.

As neoplasias mamárias observadas eram massas tumorais com superfície irregular, variando entre 2 e $10 \mathrm{~cm}$ de diâmetro. Ao corte, elas possuíam consistência firme, coloração brancacenta e estavam presentes nas regiões das glândulas mamárias torácicas e abdominais. Microscopicamente, nos adenomas foi observado epitélio glandular com arranjo tubular e na maioria havia secreção intraluminal. Já nos casos de fibroadenoma, além de a formação de túbulos, houve também proliferação do tecido fibrovascular (Figura 1A). Nos carcinomas simples tubulares, foi observada proliferação de túbulos formados por células epiteliais pleomórficas, com nucléolos evidentes (Figura 1B), sendo que em três casos foi observada acentuada proliferação de tecido fibrovascular. Havia áreas de necrose de coagulação e de uma a duas figuras de mitose por campo microscópico utilizando lente objetiva de 40x. No carcinoma mucinoso, as células tumorais se apresentaram em anel de sinete ou com citoplasma espumoso. No carcinoma simples císticopapilar, havia formação de cistos com projeções papilíferas das células epiteliais tumorais. Os aspectos macro e microscópicos dos adenomas, fibroadenomas e carcinomas simples tubulares mamários foram similares aos citados por TENNEKES et al. (2004) em ratos Wistar. Entretanto, tumores espontâneos com padrão de carcinoma mucinoso e carcinoma simples cístico-papilar constatados nos cães (MISDORP et al., 1999) não foram descritos em ratos Wistar.

Não houve casos de metástases referentes aos tumores mamários, contudo, há relatos desse fenômeno de disseminação tumoral, particularmente no tecido pulmonar (GREAVES \& FACCINI, 1992). No presente estudo, essas neoplasias ocorreram somente em fêmeas, mas já foram observadas em machos (BOMHARD \& RINKE, 1994; WALSH \& POTERACKI, 1994; POTERACKI \& WALSH, 1998). A ocorrência de tumores mamários nos ratos aumenta com a idade, havendo influência dos hormônios sexuais e ausência de envolvimento viral (CAVIGELLI et al., 2006). Dietas ricas em gordura aumentam a incidência de tumores mamários em ratos, principalmente o fibroadenoma (KRISTIANSEN et al., 1993).

Os animais com fibrossarcoma apresentaram massas tumorais aderidas à musculatura, uma de cerca de 2cm de diâmetro, localizada no membro anterior direito, e outra no focinho, com diâmetro de $3 \mathrm{~cm}$. Ao corte, elas apresentavam coloração rósea e consistência firme. Nos cortes histológicos, foram observadas células mesenquimais fusiformes, apresentando pleomorfismo, com arranjos de longos feixes entrelaçados e pouco colágeno, semelhante aos achados de WÖHRMANN \& TEREDESAI (2002). Diferente dos resultados relatados por esses autores, no presente estudo, não houve metástase. Por sua vez, os animais com hemangiossarcoma apresentavam massas circulares localizadas na pele e no tecido subcutâneo da região abdominal, coxa (direita) e membro anterior esquerdo, variando entre 4 e $10 \mathrm{~cm}$ de diâmetro. Ao corte, foram observados tecido de coloração avermelhada, consistência friável, e coágulos de sangue. Microscopicamente, eles apresentavam proliferação de espaços vasculares revestidos por células endoteliais pleomórficas e com nucléolos evidentes, algumas figuras de mitose e áreas de necrose de coagulação, conforme descreveram GREAVES \& FACCINI (1992). O animal com mesotelioma apresentava, na superfície serosa da túnica vaginal, massa de aspecto verrucoso. No corte histológico, foram observadas projeções papilíferas formadas de células epiteliais tumorais, revestindo o tecido fibrovascular, o que também foi observado por GREAVES \& FACCINI (1992) em rato.

Na glândula hipófise de três animais, foi observada massa circular de coloração avermelhada, variando de $0,5 \mathrm{~cm}$ a $1 \mathrm{~cm}$ de diâmetro (Figura 1C). No exame histológico, essas massas foram diagnosticadas como adenomas, caracterizados pela presença de células pleomórficas, com citoplasma granular, por vezes vacuolado, apresentando, em um caso, arranjo sólido (Figura 1D) e, nos outros dois, arranjo sinusóide. Foram observadas também figuras de mitose e algumas células com citoplasma apresentando grânulos castanho-amarelados. Nos animais domésticos, o adenoma pode ser grande, causando compressão do diencéfalo, e também pode possuir coloração, que varia de branco a marrom (KOESTNER et al., 1999), diferente do verificado neste estudo. O pleomorfismo celular encontrado no adenoma da glândula hipófise neste estudo, apesar de considerada uma característica de tumores malignos, é um achado comum nesse tipo de neoplasia no homem (DELELLIS et al., 2004), em ratos de laboratório (GREAVES \& FACCINI, 1992) e nos animais domésticos (KOESTNER et al., 1999). De acordo com esses autores, o carcinoma da glândula hipófise difere do adenoma por apresentar invasão nos tecidos adjacentes, metástases e acentuado pleomorfismo celular. O adenoma com produção de prolactina é o tipo de adenoma mais freqüente em ratos 

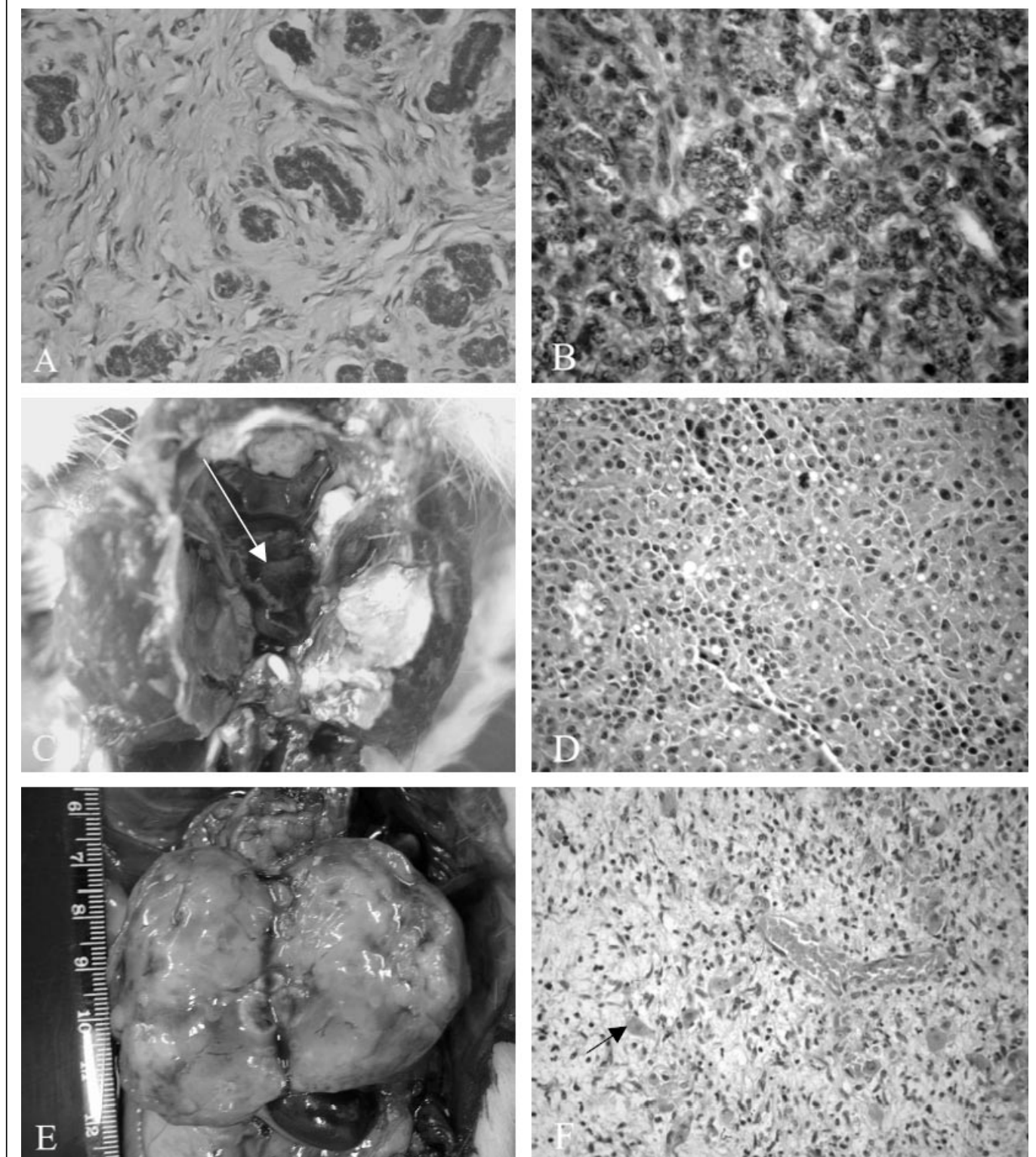

Figura 1 - A) Fotomicrografia de fibroadenoma de glândula mamária. Rato Wistar, adulto, fêmea. Proliferação celular tumoral benigna do epitélio glandular e do tecido fibrovascular. HE, obj. 20x. B) Fotomicrografia de carcinoma simples tubular de glândula mamária. Rato Wistar, adulto, fêmea. Túbulos formados por células epiteliais pleomórficas. HE, obj. 40x. C) Glândula hipófise. Rato Wistar, adulto, macho. Massa tumoral de coloração avermelhada (seta). D) Fotomicrografia de adenoma de glândula hipófise. Rato Wistar, adulto, macho. Células tumorais pleomórficas, com citoplasma granular, por vezes vacuolado, apresentando arranjo sólido. HE, obj. 40x. E) Glândula adrenal. Rato Wistar, adulto, macho. Massa tumoral de cerca de $5 \mathrm{~cm}$ de diâmetro, de aspecto gelatinoso e coloração cinza-claro. F) Fotomicrografia de ganglioneuroblastoma de glândula adrenal. Rato Wistar, adulto, macho. Células neuroblásticas imaturas, pleomórficas e em vários estágios de diferenciação, bem como células ganglionares (seta) em processo de maturação. HE, obj. 40x.

(GREAVES \& FACCINI, 1992) e no homem(DELELLIS et al., 2004). A razão para a alta prevalência de neoplasmas da glândula hipófise nos ratos é incerta (GREAVES \& FACCINI, 1992). Todavia, a redução da quantidade de ração (TUCKER, 1979) e a redução de proteína ingerida pelos animais (BERRY, 1986) podem reduzir a incidência desses tumores.

Um animal apresentou na glândula adrenal (lado direito) uma massa de cerca de $5 \mathrm{~cm}$ de diâmetro, de aspecto gelatinoso e coloração cinza-claro (Figura 
1E), que, ao exame histológico, foi diagnosticada como ganglioneuroblastoma, caracterizado pela presença de células neuroblásticas imaturas pleomórficas em vários estágios de diferenciação e de células ganglionares em processo de maturação (Figura 1F). Este é um tumor neuroblástico maligno, derivado das células neuroectodérmicas. No homem a ocorrência é maior na glândula adrenal (camada medular), mas também podem ocorrer em gânglios do sistema nervoso simpático das regiões abdominal, torácica, cervical e pélvica, sendo que aproximadamente 96\% dos casos ocorrem na primeira década da vida (KLEIHUES \& CAVENEE, 2000). BENIASHVILI \& ANISIMOV (2004) relataram casos de ratos com esse neoplasma (induzido pela administração de $\mathrm{N}$-nitrosoethylurea) no tronco simpático do mediastino, com características similares às deste estudo. No rato Wistar, o tumor na camada medular da glândula adrenal mais comum é o feocromocitoma benigno (BOMHARD \& RINKE, 1994; POTERACKI \& WALSH, 1998). Entretanto, o tumor neuroblástico mais comum que ocorre na glândula adrenal (camada medular) é o ganglioneuroma (KRINKE et al., 2000), diferente do ganglioneuroblastoma, que é um tumor benigno formado por células ganglionares maduras (BENIASHVILI \& ANISIMOV, 2004).

O animal com epitelioma sebáceo apresentou tumoração de aspecto lobulado na pele e no tecido subcutâneo da região inguinal (lado direito), esta com cerca de $2 \mathrm{~cm}$ de diâmetro e que, ao corte, possuía coloração rósea e conteúdo purulento. Microscopicamente, observou-se predomínio de células basalóides pleomórficas, poucos sebócitos, figuras de mitose e, por vezes, áreas de diferenciação em ductos, de maneira semelhante ao que GOLDSCHMIDT et al. (1998) constataram nos animais domésticos. Também foi observado infiltrado composto principalmente por neutrófilos e piócitos, confirmando a observação do conteúdo purulento na macroscopia. Ressalta-se que não foram encontrados relatos deste tumor, em ratos Wistar, na literatura consultada. Já o animal com papiloma apresentava massa tumoral na pele da cauda. O corte histológico revelou tumor benigno com proliferação de células epiteliais escamosas da pele, formando projeções papilíferas, sustentadas por tecido conjuntivo, conforme descrito por GREAVES \& FACCINI (1992) em ratos.

Macroscopicamente, o osteossarcoma osteoblástico apresentava-se como uma massa de cerca de $4 \mathrm{~cm}$ de diâmetro, localizada na face (lado esquerdo), atingindo a cavidade oral. Ao corte, ele possuía consistência firme e coloração amarelada. Microscopicamente, foram observados osteoblastos pleomórficos com nucléolos evidentes, matriz osteóide, lâminas ósseas e áreas de necrose de coagulação, que compreendem características histológicas também relatadas por GREAVES \& FACCINI (1992) em ratos e por SLAYTER et al. (1994) nos demais animais domésticos.

O animal com leiomioma apresentava massa branca, de consistência firme, e de cerca de $1 \mathrm{~cm}$ de diâmetro no corno uterino esquerdo. No exame microscópio, foram observados feixes entrelaçados de células musculares lisas tumorais com características benignas, sendo que tais observações estão de acordo com as descrições de GREAVES \& FACCINI (1992) em ratos. Pela coloração Fucsina Ácida-Van Gieson, houve o predomínio da coloração amarela, indicando que o tumor era constituído por fibras musculares.

No animal com adenocarcinoma papilífero, havia um nódulo de consistência endurecida e cor esbranquiçada, medindo cerca de $3 \mathrm{~mm}$ de diâmetro no parênquima pulmonar. Na análise do corte histológico, observou-se proliferação de células epiteliais colunares pleomóficas, apresentando arranjos papilíferos, sendo tais achados condizentes com adenocarcinoma papilífero pulmonar primário. O tumor foi classificado de acordo como o proposto por GREAVES \& FACCINI (1992) em ratos e DUNGWORTH et al. (1999) nos animais domésticos.

\section{CONCLUSÕES}

Neste estudo a prevalência de neoplasias espontâneas nos ratos foi bastante inferior à encontrada em outras pesquisas. Houve grande variedade quanto aos tipos de neoplasias, entretanto, predominaram os tumores mamários, com maior ocorrência dos carcinomas, sendo que estes foram seguidos do fibroadenoma. Os tumores malignos foram os mais freqüentes e os animais mais acometidos eram fêmeas e adultos. Este artigo é pioneiro na descrição do epitelioma sebáceo e dos carcinomas mamários mucinoso e simples cístico-papilar de ocorrência espontânea em ratos Wistar.

\section{AGRADECIMENTOS}

À Dra. Luciana Wernersbach Pinto, médica patologista do Instituto de Pesquisa Evandro Chagas (IPEC), Fundação Oswaldo Cruz (FIOCRUZ), Rio de Janeiro, pelo auxílio nos diagnósticos, e à Dra. Belmira Ferreira dos Santos, médica veterinária do CECAL, FIOCRUZ, pelo incentivo, pelas informações e pelo fornecimento dos animais.

\section{COMITÊ DE ÉTICA E BIOSSEGURANÇA}

O desenvolvimento deste trabalho foi autorizado pelo Comitê de Ética para o Uso de Animais (CEUA/ FIOCRUZ), por meio da aprovação do programa n P0044-00. 


\section{REFERÊNCIAS}

BENIASHVILI, D.S.; ANISIMOV, V.N. Morphology of experimental induced tumors of the sympathetic nervous system in rats. Experimental and Toxicology Pathology, v.56, n.1-2, p.53-58, 2004.

BERRY, P.H. Effect of diet or reproductive status on the histology of spontaneous pituitary tumors in female Wistar rats. Veterinary Pathology, v.23, n.5, p.610-618, 1986.

BOMHARD, E.; RINKE, M. Frequency of spontaneous tumours in Wistar rats in 2-year studies. Experimental and Toxicologic Pathology, v.46, n.1, p.17-29, 1994.

CAVIGELLI, S.A. et al. Infant temperament predicts life span in female rats that develop spontaneous tumors. Hormones and Behavior, v.50, n3, p. 454-462, 2006.

DELELLIS, R.A. et al. Pathology and Genetics of Tumors of endocrine organs. Lyon: IARC, 2004. V.8, 320p.

DUNGWORTH, D.L. et al. World Health Organization. Histological classification of tumors of the respiratory system of domestic animals. 2.ed. Washington: Armed Forces Institute of Pathology and American registry of Pathology, 1999. 70p.

GOLDSCHMIDT, M.H. et al. World Health Organization. Histological classification of epithelial and melanocytic tumors of the skin of domestic animals. 2.ed. Washington: Armed Forces Institute of Pathology and American registry of Pathology, 1998. 105p.

GREAVES, P.; FACCINI, J.M. Rat histopatology. 2.ed. Amsterdam: Elsevier, 1992. 302p.

HARKNESS, J.E.; WAGNER, J.E. Biologia e clínica de coelhos e roedores. 3.ed. São Paulo: Roca, 1993. 238p.

HENDRICK, M.J. et al. World Health Organization. Histological classification of mesenchymal tumors of skin and soft tissues of domestic animals. 2.ed. Washington: Armed Forces Institute of Pathology and American registry of Pathology, 1998. 62p.

KENNEDY, P.C. et al. World Health Organization. Histological classification of tumors of the genital system of domestic animals. 2.ed. Washington: Armed Forces Institute of Pathology and American registry of Pathology, 1998. 78p.

KLEIHUES, P.; CAVENEE, W.K. Pathology and genetics of tumors of the nervous system. Lyon: IARC, 2000. V.1, 314p.
KOESTNER, A. et al. World Health Organization. Histological classification of tumors of the nervous system of domestic animals. 2.ed. Washington: Armed Forces Institute of Pathology and American registry of Pathology, 1999. 71p.

KOHN, D.F.; BARTHOLD, S.W. Biology and diseases of rats. In: FOX, J.G. et al. Laboratory animal medicine. San Diego: Academic, 1984. p. 91-122.

KRINKE, G.J. et al. Morphologic characterization of spontaneous nervous system tumors in mice and rats. Toxicologic Pathology, v.28, n.1, p.178-192, 2000.

KRISTIANSEN, E. et al. Effects of high-fat diet on incidence of spontaneous tumors in Wistar rats. Nutrition and Cancer, v.19, n.1, p.99-110, 1993.

MISDORP, W. et al. World Health Organization. Histological classification of mammary tumors of the dog and cat. 2.ed. Washington: Armed Forces Institute of Pathology and American registry of Pathology, 1999. 59p.

PECKHAM, J.C. Experimental oncology. In: BAKER, H.J. et al. The laboratory rat: research applications. San Diego: Academic, 1980 . p.119-147.

POTERACKI, J.; WALSH, K.M. Spontaneous neoplasms in control Wistar rats: a comparison of reviews. Toxicological Sciences, v.45, n.1, p.1-8, 1998.

SLAYTER, M.V. et al. World Health Organization. Histological classification of bone and joint tumors of domestic animals. 2.ed. Washington: Armed Forces Institute of Pathology and American registry of Pathology, 1994. 50p.

TENNEKES, H. et al. The stability of historical control data for common neoplasms in laboratory rats: adrenal gland (medulla), mammary gland, liver, endocrine pancreas, and pituitary gland. Regulatory Toxicology and Pharmacology, v.40, n.1, p.18-27, 2004.

TUCKER, M.J. The effect of long-term food restriction on tumors in rodents. International Journal of Cancer, v.23, n.6, p.803-807, 1979.

WALSH, K.M.; POTERACKI, J. Spontaneous neoplasms in control Wistar rats. Fundamental and Applied Toxicology, v.22, n.1, p.65-72, 1994.

WÖHRMANN, T.; TEREDESAI, A. Metastasizing fibrossarcoma in a Wistar rat-Case report. Journal of Veterinary Medicine Series A, v.49, n.10, p.538-540, 2002. 